\title{
CALCULATION OF THE THERMAL DYNAMIC PERFORMANCE OF THE RESIDENTIAL BUILDINGS' WALLS
}

doi: 10.2478/cqpi-2019-0029

Date of submission of the article to the Editor: 15/03/2019

Date of acceptance of the article by the Editor: 12/05/2019

\author{
Saša M. Kalinović ${ }^{1}$ \\ Jelena M. Djoković ${ }^{1}$ - orcid id: 0000-0001-5542-8088 \\ Ružica R. Nikolić ${ }^{2}$ - orcid id: 0000-0003-3042-8916 \\ Branislav Hadzima ${ }^{2}$ - orcid id: 0000-0002-8201-905X \\ 1 University of Belgrade, Serbia \\ ${ }^{2}$ University of Žilina, Slovakia
}

\begin{abstract}
Calculation of the thermal dynamic properties of the multi-layer wall isolation of residential buildings is presented in this paper. Taking into account that the final objective is to create a building with the highest energy efficiency ratio, i.e. with the lowest energy consumption, both for heating and cooling, it is necessary to realize the good thermal characteristics of the multi-layer wall. To obtain the optimal solution for the wall's structure, various wall structures with different thicknesses of the individual layers, were analyzed. Based on results, presented in this paper, one can conclude that for walls with the same total thickness, but various thicknesses of the individual layers, that constitute the complete wall structure, the differences appear in the delay of the change of the walls outside temperature. In that way, by varying those individual layers' thicknesses, one can obtain the optimal solution for the wall structure with the highest savings of energy.
\end{abstract}

Keywords: wall's specific mass, heat conduction coefficient, periodical heat transfer, reduction factor, thermal inertia.

\section{INTRODUCTION}

According to the newest data, published by the US Energy International Administration, energy consumptions in buildings represents $36 \%$ of the total world energy consumption, where the largest share of $26 \%$ is assigned to residential buildings (EIA, 2016). Investigations done by this agency show that facilities providing energy for residential building are responsible for about $30 \%$ of the world's harmful gasses emission producing the green-house effect. Bearing those investigations in mind, one can conclude that the buildings are the biggest individual energy consumers, with tendency of the consumption increase due to the world's population growth. This is why the buildings' energy efficiency increase makes a large contribution to reducing the energy consumption and environment pollution. 
Energy needs of a building depend on the structure of the thermal envelope, building's geometry, its exposure to the Sun and winds, thermal characteristics of materials from which the building is built of, as well as of windows and doors tightness. Besides of these factors, there are numerous physical parameters which reflect the dynamic thermal characteristics of materials. Those are the parameters that influence the possibility for the heat accumulation, like the specific heat, thermal inertia, material's density (specific mass) and parameters that affect the heat conduction rate - thermal diffusivity, heat time constant. All of those parameters directly affect the thermal comfort in the building, as well as calculation of its energy needs, while they generally defined as the constant values. However, the buildings are exposed to variable weather conditions, like the temperature variation on the daily, season, yearly or multiyearly levels, variations in the quantity of humidity in the air, the Sun radiation, presence of various particles or compounds in the air, etc. Characteristics of the builtin materials and their physical properties during the exploitation of objects are not constant, they have the tendency of natural ageing, as shown in (Giarma and Kontoleon, 2016) and (Kosnyb and Kosecka, 2002). Estimate of thermal and physical characteristics of the built-in materials and their changes during the exploitation are important for evaluation of the energy performance of the object itself, as well as for estimate of the object's heat losses, (Koo et al., 2015).

The value of the heat conduction coefficient, obtained based on calculations in the stationary heat conduction conditions, is not an adequate indicator of the thermal behavior of the building elements, since it is possible that two walls, having the same value of the heat conduction coefficient, absorb or release the heat at different rates, (McMullan, 2018). The reason for that lies in the fact that the influence of the heat capacity is being neglected in the stationary heat conduction conditions and analysis is being conducted based on the material's heat conduction, only. Variation of number of people that are present in the building, of heating and cooling, all of those in combination with variation of the outside temperature, are causing that the building is frequently in the state of flux and that it is actually necessary to estimate the dynamic behavior of the whole building during the determination of the optimal materials for the building's envelope. Material characteristics, like specific heat capacity $-c$, density $-\rho$ and the heat conductivity $-\lambda$, play the very important role in dynamic behavior of a structure, which is strongly expressed when the outside temperature cyclically changes with respect to the desired internal temperature. Materials with the useful thermal properties are the insulating materials or materials with the thermal mass. Influences of the thermal mass and the thermal insulation are simultaneously taken into account during the checking of dynamic and stationary thermo-mechanical characteristics of materials, (Nicol and Humphreys, 2002).

Materials of the large thermal mass, exposed to the heat source, can store more heat as compared to other materials. They also release the accumulated heat much slower, once the heat source is gone, (Gregory et al., 2008). During the winter days, materials with the large thermal mass can store the heat from the incoming Sun radiation and then to release it into the interior of the room, later during the evening hours, when the passive heat source is removed and the higher inside temperature is required. In such a way the mechanical heating of the room is reduced. On the other hand, during the summer days, the thermal mass lowers the temperature of the interior room caused by the Sun radiation, preventing the appearance of "peaks" of the inside temperature and increased loading of the air-conditioners. 


\section{$\underline{214}$}

Since the building elements are storing the heat during the day, later in the evening they will release it, partially into the interior of the room and partially into the environment. Into the interior, due to sufficient time delay, the heat can be transferred by using the cooler outside air via the natural ventilation, while emission into the environment can be accelerated by the so-called radiation of the clear skies. The bigger difference in temperatures of the warmer interior and cooler environment improves this filtering process, (Balaras, 1996). The heat insulator slows down the heat transfer between different zones at different temperatures and limits the heat loss through the construction material during the winter period and heat flow into the interior during the summer (Kruger et al., 2010).

The dynamic thermal performance of buildings is becoming lately the subject of research of numerous investigators. Mohammad and Shea (2013) have analyzed the thermal performance of the wall materials used in residential buildings in Tehran. The best and the worst types of buildings were determined through the comparison of the predicted inside temperature and targeted temperature of the thermal comfort. Brambilla et al. (2018) presented a preliminary study of influence of the thermal mass and ventilation on the thermal comfort in standard business buildings in Fribourg, Switzerland, which are marked as the smart building. Analysis has included various levels of the thermal mass, types of structures, ventilation strategies and several risk scenarios, where the standard assumptions of the heat simulation were changed in order to account for critical situation. Results have pointed to importance of the natural ventilation as the principle of the passive cooling, regardless of the type of structure. In addition, they also show that the thermal mass is necessary for stabilizing the inside temperatures, and that its potential came to light only in combination with natural ventilation. Those results have shown that the thermal mass per se is not sufficient for providing the required thermal comfort. Albatayneh et al. (2019) have recorded the inside air temperatures for more than a year of heating or cooling, then the number of hours needed for heating or cooling calculated based on the fixed sets of the working temperatures and model of the adaptive thermal comfort, in order to estimate the number of hours needed for cooling and heating to provide the thermal comfort of residents for the four tested residential areas in the campus of the Newcastle University in Australia. It is shown that the adaptive thermal comfort model significantly reduces time needed for mechanical cooling and heating, even for more than a half in comparison to adjusting the thermostats, which are used in the existing air-conditioning systems. The furniture and other interior objects can impose the significant influence on the interior heat dynamics and residential comfort (Johra and Heiselberg, 2017). The representative values of the furniture parameters are presented in this paper, namely the interior thermal masses, obtained by application of various models. It was concluded that the large exposed areas of furniture can significantly increase the effective thermal inertia of the building without any construction works. Ferrariand and Zanotto (2016) have shown that the non-stationary processes of the heat exchange between the building and environment, i.e. the calculation of the object's dynamic behavior, present the real estimate of its thermal performances. The method, presented in their paper introduces the new parameters into the thermal analysis: the heat capacity of the envelope's inertia, the phase shift of the outside temperature oscillations and the reduction factor of these oscillations.

The heat dynamic characteristics of certain walls are analyzed in this paper in order to come up with the best (optimal) solutions for the residential objects in Serbia, which 
would satisfy the prescribed laws and requirements defined by (Rules on the energy efficiency of the building, 2011).

\section{PROBLEM FORMULATION}

In Figure 1 is presented the process of the heat conduction through the flat homogeneous wall in the wall thickness direction in the stationary conditions, Figure 1 (a) and in the non-stationary conditions, Figure 1(b). In the stationary conditions, the temperature difference at the wall surfaces is constant, while the temperature profile within the wall thickness does not vary with time. The amount of heat that enters the wall is equal to the amount of heat that exits for the wall. In the non-stationary conditions, the temperature profile within the wall is variable with time, while the amount of heat that enters the wall is not equal to the amount of heat that exits from the wall, since the part of the heat is accumulated in the wall.

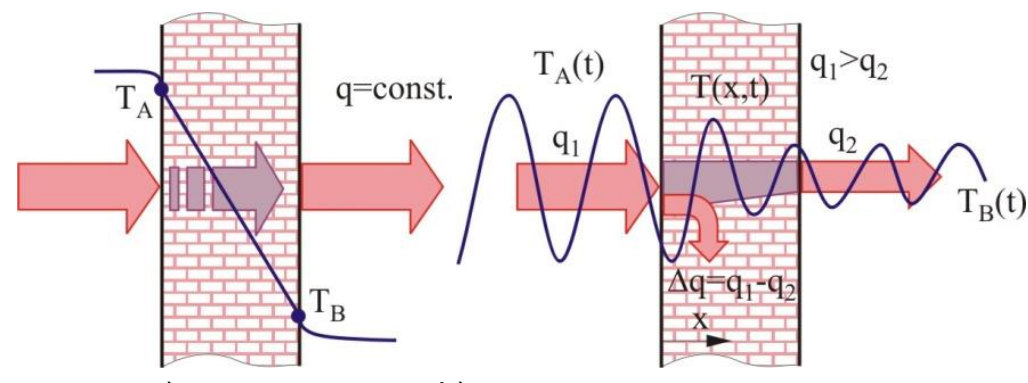

a)

b)

Fig. 1. Schematic presentation of the heat conductions through the flat homogeneous wall: a) stationary conditions, b) non-stationary conditions.

Equation of the heat conduction in the wall thickness direction, when there is no heat generated within the wall, for the non-stationary conditions, reads:

$$
a \frac{\partial^{2} T}{\partial x^{2}}=\frac{\partial T}{\partial \tau}
$$

where the variable $a=\lambda / \rho c\left[\mathrm{~m}^{2} / \mathrm{s}\right]$ represents the heat diffusivity of the wall material and it characterizes the heat diffusion through the given material.

The relation between the complex temperature amplitude and the flux on surfaces of the homogenous, single-layer wall, in the matrix form is (EN ISO 13786):

$$
\left(\begin{array}{l}
\hat{T}_{w e}(j \omega) \\
\hat{q}_{w e}(j \omega)
\end{array}\right)=\left[\begin{array}{ll}
Z_{11} & Z_{12} \\
Z_{21} & Z_{22}
\end{array}\right]\left(\begin{array}{l}
\hat{T}_{w i}(j \omega) \\
\hat{q}_{w i}(j \omega)
\end{array}\right)
$$

where $\hat{q}_{w i}$ and $\hat{q}_{w e}$ are the heat fluxes, in the complex domain, at the inside and outside wall surfaces, respectively, $\hat{T}_{w i}$ and $\hat{T}_{w e}$ are the temperature amplitudes, in the complex domain, at the inside and outside wall surfaces, respectively, while $Z_{n m}$ ( $n, m$ $=1,2$ ), elements of the wall transfer matrix, with characteristics $\lambda, \rho, c$ and $d$, which relates the temperature and the heat flux at one side of the wall to the same variables at the other side, have the following form:

$$
Z_{11}=Z_{22}=\cosh (\xi+j \cdot \xi)=\cosh (\xi) \cosh (j \cdot \xi)+\sinh (\xi) \sinh (j \cdot \xi)
$$


$\underline{216}$

$$
\begin{aligned}
& Z_{12}=-\frac{\delta}{2 \lambda}[(\sinh (\xi) \cos (\xi)+\cosh (\xi) \sin (\xi))+j \cdot(\cosh (\xi) \sin (\xi)-\sinh (\xi) \cos (\xi))](4) \\
& Z_{21}=-\frac{\lambda}{\delta}[(\sinh (\xi) \cos (\xi)-\cosh (\xi) \sin (\xi))+j \cdot(\sinh (\xi) \cos (\xi)+\cosh (\xi) \sin (\xi))]
\end{aligned}
$$

where:

$$
\delta=\sqrt{\frac{\lambda T}{\pi \rho c}} \text { and } \xi=\frac{d}{\delta}
$$

and where $d$ is the wall thickness.

System of equations (2), with matrix elements defined by equations (3) to (5), relates temperatures and heat fluxes, i.e. their corresponding amplitudes, from one side of the wall to the same variables at the other side of the wall, at frequency $\omega$. Each element $Z_{n m}$ is a complex number, which can be represented by its module $\left|Z_{n m}\right|$ and argument $\varphi_{n m}=\arg \left(Z_{n m}\right)$. Elements $Z_{n m}$ of the heat transfer matrix in system (2) have the following physical meaning: $\left|Z_{11}\right|$ is the temperature amplitude factor, i.e. the temperature variation amplitude at the external side of the wall when the temperature variation at the internal side of the wall is $1[\mathrm{~K}]$ and the heat flux at the internal side of the wall is constant; $\varphi_{11}$ is the phase shift between the temperatures on two sides of the wall; $\left|Z_{21}\right|$ represents the amplitude of the heat flux variation at the external side of the wall when the temperature variation at the internal side of the wall is $1[\mathrm{~K}]$ and the heat flux at the internal side of the wall is constant; $\varphi_{21}$ is the phase shift between heat flux at the external side of the wall and temperature at the internal side of the wall; $\left|Z_{12}\right|$ represents the amplitude of the temperature variation at the external side of the wall when the heat flux variation at the internal side of the wall is $\left[1 \mathrm{~W} / \mathrm{m}^{2}\right]$ and the temperature at the internal side of the wall is constant; $\varphi_{12}$ is the phase shift between the temperature at the external side of the wall and the heat flux at the internal side of the wall; $\left|Z_{22}\right|$ is the heat flux amplitude factor, i.e. amplitude of the heat flux variation at the external side of the wall when the heat flux variation amplitude at the internal side of the wall and the temperature at the internal side of the wall is constant; $\varphi_{22}$ is the phase shift between the heat fluxes at the two sides of the wall.

The $Z_{n m}$ elements are used for calculating the thermal transmittance and thermal admittance of the wall. The thermal admittance of the wall relates the heat flux at one side of the wall to temperature variation at the same side of the wall, while the thermal transmittance defines the relation of certain physical variables from one and the other side of the wall.

Heat flux variation at the internal side of the single-layer, homogeneous facade wall, of the thickness $d$, of infinite area, in the time domain, when the temperature excitation at the wall surface is the Dirac $\delta$ function, is:

$$
q_{w i, \delta}(t)=\sum_{k=1}^{\infty}-4 \frac{\lambda^{2}}{\rho c d^{3}}(2 k-1)^{2} \pi^{2} e^{-\frac{\lambda(2 k-1)^{2} \pi^{2}}{\rho c d^{2}} t}=-q_{w e, \delta}(t)
$$

Solution for the external side of the wall is identical to solution of (7) only with a minus sign.

Equation (7) is valid only for homogeneous single-layer walls. However, walls on building are mainly multi-layer and heterogeneous, so the given solutions do not apply, but the solution for the heterogeneous multi-layer wall can be derived from 
them. Writing the matrix equations (7) for each layer of the wall $i, \quad i=\overline{1, n}$, and setting that the limit values between the layer $i$ and layer $i+1$ are equal, the matrix equation (2), for the heterogeneous wall of $n$ layers, can be written as:

$$
\left(\begin{array}{l}
\hat{T}_{w e} \\
\left.\hat{q}_{w e}\right)
\end{array}\right)=\left[\begin{array}{ll}
Z_{N, 11} & Z_{N, 12} \\
Z_{N, 21} & Z_{N, 22}
\end{array}\right]\left(\begin{array}{l}
\hat{T}_{w i} \\
\hat{q}_{w i}
\end{array}\right)
$$

Where $n$ is the number of wall's layers. The thermal and physical parameters $\lambda_{i}, \rho_{i}, c_{i}$ as well as the thickness $d_{i}$, are defined for each layer. Counting of layers starts from the internal side.

The matrix elements $Z_{N, n m}$ define the thermal dynamic parameters of the wall, which describes the time-dependent processes occurring in the wall. Those parameters are: $Y_{11 / 22}\left[\mathrm{~W} / \mathrm{m}^{2} \mathrm{~K}\right]$ - internal and external thermal admittance, $Y_{12}\left[\mathrm{~W} / \mathrm{m}^{2} \mathrm{~K}\right]$ - periodical thermal transmittance, $f[-]$ - decrement factor, $T_{11 / 22}[\mathrm{~h}]$ - phase shift of the thermal admittance, $T_{12}[\mathrm{~h}]$ - phase shift of thermal transmittance, $k_{1 / 2}\left[\mathrm{~J} / \mathrm{m}^{2} \mathrm{~K}\right]$ - surface thermal capacities.

The thermal admittance is a complex variable that represents the ratio of the complex amplitude of the heat flux variation at one side of the wall and complex amplitude of the temperature variation at the same side of the wall, when the temperature at the other side of the wall is constant. It is calculated according to the following expressions:

$$
Y_{11}=-\frac{Z_{11}}{Z_{12}} Y_{22}=-\frac{Z_{22}}{Z_{12}}
$$

The periodical thermal transmittance is a complex variable that represents the ratio of the complex amplitude of the heat flux variation at one side of the wall and the complex amplitude of the temperature variation at the other side of the wall, when the temperature at that side of the wall is constant. It is calculated as:

$$
Y_{12}=-\frac{1}{Z_{12}}
$$

The decrement factor represents the ratio of the periodical thermal transmittance module and the stationary thermal transmittance, namely the heat conduction coefficient, i.e.

$$
f=\frac{\left|Y_{12}\right|}{U}
$$

The decrement factor value represents the degree of damping the wall temperature amplitude at the external side of the wall with respect to the temperature amplitude at the internal side of the wall. The value of this factor ranges between 0 and 1 . If its value is closer to 0 it means that the wall has strong damping and that is the characteristics of the massive structures.

The phase shift of the periodical thermal transmittance and thermal admittance represents the period for which the temperature amplitude or the heat flux amplitude passes through the structural element; thou are being defined in the following way:

$$
\tau_{12}=\frac{T}{2 \pi} \arg \left(-1 / Z_{12}\right) \tau_{11 / 22}=\frac{T}{2 \pi} \arg \left(Y_{11 / 22}\right)
$$

respectively. 


\section{$\underline{218}$}

As in the case of the decrement factor, the lighter structures have the shorter phase shift compared to the massive ones.

The surface thermal capacities represent the thermal capacities per unit area of the structural element; they are defined as:

$$
k_{1}=\frac{T}{2 \pi}\left|\frac{Z_{11}-1}{Z_{12}}\right| k_{2}=\frac{T}{2 \pi}\left|\frac{Z_{22}-1}{Z_{12}}\right|
$$

Taking into account that in all the equations appear the elements of the transfer matrix $Z_{n m}$, calculation of all the aforementioned parameters requires knowing the thermal characteristics of each wall's layer $\lambda_{i}, \rho_{i}, c_{i}$ as well as its thickness $d_{i}$.

\section{RESULTS AND DISCUSSION}

Here are analyzed dynamic performances of the heat conduction for various wall constructions.

The calculation procedure of dynamic characteristics of walls, namely variables that define the dynamic behavior of walls include: identifying the materials that make the layers of the building structure, thicknesses of all these layers as well as the thermal characteristics of those materials, determination of the period of the temperature variations on surfaces, calculation of the heat penetration depth for each layer, determination of the transfer matrix for each layer (component), multiplication of the transfer matrix of each layer, excluding those peripheral layers, in the proper order for obtaining the transfer matrix of the wall.

Three types of walls are considered in this research. They have the same total thickness, but different thicknesses of individual layers that constitute the complete wall structure. In these three cases the thermo-insulating layer occupied different positions, as well.

In Figure 2 are shown three types of walls of similar structures, considered in these calculation.

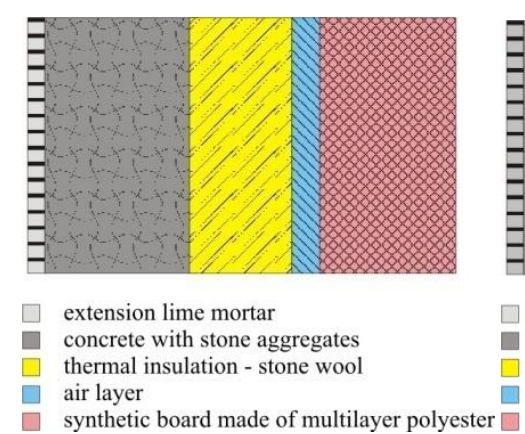

(a)

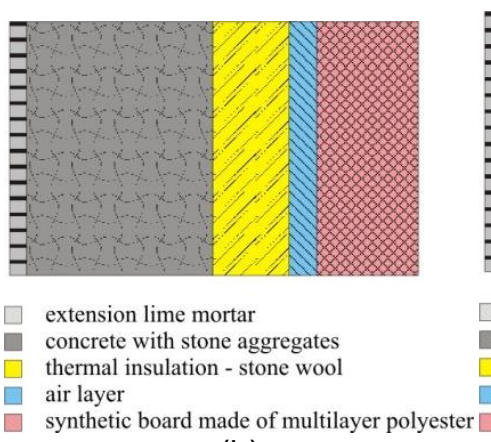

(b)

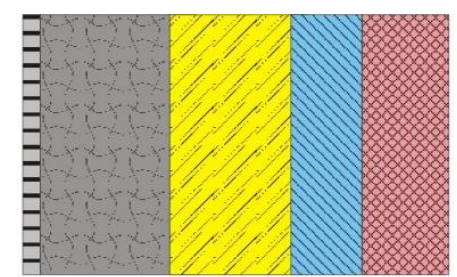

extension lime mortar

concrete with stone aggregates

thermal insulation - stone woo air layer

synthetic board made of multilayer polyester (c)

Fig. 2. Structures of the considered walls: a) wall 1, b) wall 2 , c) wall 3.

Structures of the considered walls are shown in Table 1. 
Table 1. Structures of the considered walls

\begin{tabular}{|l|l|l|l|}
\hline \multirow{2}{*}{ Wall structure } & \multicolumn{3}{|c|}{ Wall layer thickness [m] } \\
\cline { 2 - 5 } & Wall 1 & Wall 2 & Wall 3 \\
\hline Extension lime mortar & 0.015 & 01 & 0.015 \\
\hline Concrete with stone aggregates & 0.15 & 0.2 & 0.1 \\
\hline Thermal insulation-stone wool & 0.1 & 08 & 0.150 \\
\hline Air layer & 0.04 & 0.035 & 0.08 \\
\hline Synthetic board made of multilayer polyester & 0.12 & 0.1 & 0.08 \\
\hline
\end{tabular}

Basic characteristics of walls presented in Figure 2 are given in Table 2.

Table 2. Basic characteristics of the considered walls

\begin{tabular}{|l|l|l|l|}
\hline & Wall 1 & Wall 2 & Wall 3 \\
\hline Thickness $[\mathrm{m}]$ & 0.425 & 0.425 & 0.425 \\
\hline Heat conduction coefficient, $U\left[\mathrm{~W} / \mathrm{m}^{2} \mathrm{~K}\right]$ & 0.197 & 0.233 & 0.13 \\
\hline Mass $\left[\mathrm{kg} / \mathrm{m}^{2}\right]$ & 576 & 642 & 460 \\
\hline
\end{tabular}

Dynamic characteristics of the considered walls are shown in Figure 2 and Table 3.

Table 3. Dynamic characteristics of the considered walls

\begin{tabular}{|l|l|l|l|}
\hline Dynamic characteristics & Wall 1 & \multicolumn{1}{|c|}{ Wall 2 } & Wall 3 \\
\hline Internal thermal admittance, $Y_{11}\left[\mathrm{~W} / \mathrm{m}^{2} \mathrm{~K}\right]$ & $\begin{array}{l}5.572 \\
(1.01 \mathrm{~h})\end{array}$ & $\begin{array}{l}5.482 \\
(0.92 \mathrm{~h})\end{array}$ & $\begin{array}{l}5.733 \\
(1.35 \mathrm{~h})\end{array}$ \\
\hline External thermal admittance, $Y_{22}\left[\mathrm{~W} / \mathrm{m}^{2} \mathrm{~K}\right]$ & $\begin{array}{l}4.739 \\
(2.46 \mathrm{~h})\end{array}$ & $\begin{array}{l}4.78 \\
(2.43 \mathrm{~h})\end{array}$ & $\begin{array}{l}4.916 \\
(2.45 \mathrm{~h})\end{array}$ \\
\hline Periodical thermal transmittance, $Y_{12}\left[\mathrm{~W} / \mathrm{m}^{2} \mathrm{~K}\right]$ & $\begin{array}{l}0.003 \\
(1.17 \mathrm{~h})\end{array}$ & $\begin{array}{l}0.005 \\
(2.71 \mathrm{~h})\end{array}$ & $\begin{array}{l}0.001 \\
(-1.42 \mathrm{~h})\end{array}$ \\
\hline $\begin{array}{l}\text { Thermal capacity of the heat surface at the internal } \\
\text { side, } k_{1}\left[\mathrm{~kJ} / \mathrm{m}^{2} \mathrm{~K}\right]\end{array}$ & 76.6 & 75.3 & 78.8 \\
\hline $\begin{array}{l}\text { Thermal capacity of the heat surface at the } \\
\text { external side } k_{2}\left[\mathrm{~kJ} / \mathrm{m}^{2} \mathrm{~K}\right]\end{array}$ & 65.1 & 65.7 & 67.6 \\
\hline Decrement factor, $f[-]$ & 0.015 & 0.02 & 0.01 \\
\hline Factor of the temperature variation shift, $\tau_{12}[\mathrm{~h}]$ & 22.83 & 21.29 & 1.42 \\
\hline
\end{tabular}

Based on results presented in Table 3 one can notice that the differences of the heat conduction coefficient between walls 1 and 2 are almost twice smaller than those between walls 2 and 3, while the specific masses of walls are significantly different.

It can also be seen that the phase shift at the external and internal sides of the wall is approximately equal for all the walls, with the difference between walls 1 and 2 being slightly smaller. This means that walls react to change of the outside temperature practically at the same rate.

The thermal capacities of the wall surface at the external side has the largest value for wall 3 , while the values for walls 1 and 2 are smaller and approximately the same $\left(65.1 \mathrm{~kJ} / \mathrm{m}^{2} \mathrm{~K}\right.$ and $65.7 \mathrm{~kJ} / \mathrm{m}^{2} \mathrm{~K}$, respectively).

The reduction factor of the external temperature variation is the largest for wall 2 (0.02), wall 1 has for $25 \%$ smaller value than the wall 2 , while wall 3 has twice smaller value (0.01). 


\section{$\underline{220}$}

The consequence of all the mentioned is that the shift in the external temperature variation for the wall 3 is about 16 times smaller $(1.42 \mathrm{~h})$ then in walls $1(22.83 \mathrm{~h})$ and $2(21.29 \mathrm{~h})$.

\section{CONCLUSIONS}

In this paper are analyzed non-stationary processes of the heat exchange between the building and environment, namely the dynamic thermal behavior of a building in order to realistically estimate its thermal performances. The heat inertia of the thermoinsulating and painting materials of the building's envelope was used to describe this phenomenon. It describes the ability of a material or a structure to store the thermal energy and delays its transfer.

The thermal capacity of the massive wall structure elements is defined by the phase shift. The ability of the temporary delay of the external thermal effect was analyzed, together with factors for reduction of the external temperatures at the internal side of the wall, as an essential dynamic characteristics of a wall. It is shown that obtaining the adequate values of the heat conduction coefficient of the wall is related to its structure.

Three types of walls were analyzed in the paper in order to reach the optimal solution of the wall structure with highest potential for energy savings. The three considered walls have the same total thickness, but the wall's constitutive layers have different thicknesses.

Based on results presented in this paper one can conclude that in walls of similar structures and the same thickness, there appear differences in the external temperature variation shift due to the different thickness of the individual layers constituting the walls structure.

The general conclusion is that more attention should be paid to study and research of the dynamic thermal performances of various wall structures and that the tendency should be towards obtaining the optimal solutions which would provide for the energy savings, while simultaneously satisfying the legal regulations. Such solutions would be economically sustainable while providing the best possible residential comfort to buildings' occupants.

\section{ACKNOWLEDGEMENTS}

This research was partially financially supported by the Ministry of Education, Science and Technological Development of Republic of Serbia through grants ON 174001 and ON 174004 and by European regional development fund and Slovak state budget by the projects ITMS2014+313011D011 "Research Centre of the University of Žilina" and NFP313010T426 "Research and development activities of Žilina University in Žilina for the 21 st century industry in the field of materials and nanotechnologies".

\section{REFERENCES}

Albatayneh, A., Alterman, D., Page, A., Moghtaderi, B., 2019. The Significance of the Adaptive Thermal Comfort Limits on the Air-Conditioning Loads in a Temperate Climate. Sustainability, 11, 328-345.

Balaras, C. A., 1996. The role of thermal mass on the cooling load of buildings: An overview of computational methods. Energy and Buildings, 24, 1-10. 
Brambilla, A., Bonvin, J., Flourentzou, F., Jusselme, T., 2018. On the Influence of Thermal Mass and Natural Ventilation on Overheating Risk in Offices. Buildings, 8, 47-58.

EIA (US Energy Information Administration), 2016. International Energy Outlook 2016 With Projections to 2024. Washington, DC, US. https://www.eia.gov/outlooks/ieo/

EN ISO 13786 Standard 2017: Thermal performance of building components -- Dynamic thermal characteristics - Calculation methods

Ferrariand S., Zanotto, V., 2016. Building Energy Performance Assessment in Southern Europe. Springer, Berlin, Heidelberg, Leipzig, Germany.

Giarma, C., Kontoleon, K. J., 2016. Dynamic thermal response of building material layers in aspect of their moisture content. Applied Energy, 170, 76-91,

Gregory, K., Moghtaderi, B., Sugo, H., Page, A., 2008. Effect of thermal mass on the thermal performance of various Australian residential constructions systems, Energy and Buildings, 40, 459-465.

Johra, H., Heiselberg, P., 2017. Influence of internal thermal mass on the indoor thermal dynamics and integration of phase change materials in furniture for building energy storage: A review. Renewable and Sustainable Energy Reviews, 69, 19-32.

Koo, C., Kim, J., Lee, M., Jeong, K., Hong, T., 2015. A review on sustainable construction management strategies for monitoring, diagnosing, and retrofitting the building's dynamic energy performance: Focused on the operation and maintenance phase. Applied Energy, 155.

Kosnyb, J., Kossecka, E., 2002. Influence of insulation configuration on heating and cooling loads in a continuously used building. Energy and Buildings, 34, 321-331.

Kruger, E., Cruz, E.G., Givoni, B., 2010. Effectiveness of indirect evaporative cooling and thermal mass in a hot arid climate. Building and Environment, 45, 1422-1433.

McMullan, R., 2018. Environmental Science in Buildings. Palgrave Macmillan Education, London, UK.

Mohammad, S., Shea, A., 2013. Performance Evaluation of Modern Building Thermal Envelope Designs in the Semi-Arid Continental Climate of Tehran. Energy and Buildings, 3, 674-688.

Nicol, J. F., Humphreys, M.A., 2002. Adaptive thermal comfort and sustainable thermal standards for buildings. Energy and Buildings, 34, 563-572.

Rules on the energy efficiency of the building, 2011. Official Gazette of Republic of Serbia, 11/2011. 